\title{
Traffic Swarm Behaviour: Machine Learning and Game Theory in Behaviour Analysis
}

\author{
Gergely Hollósi, Csaba Lukovszki, Máté Bancsics, and Gábor Magyar
}

\begin{abstract}
High density traffic on highways and city streets consists of endless interactions among participants. These interactions and the corresponding behaviours have great impact not only on the throughput of traffic but also on safety, comfort and economy. Because of this, there is a great interest in deeper understanding of these interactions and concluding the impacts on traffic participants. This paper explores and maps the world of traffic behaviour analysis, especially researches focusing on groups of vehicles called traffic swarm, while presents the stateof-the-art methods and algorithms. The conclusion of this paper states that there are special areas of traffic behaviour analysis which have great research potential in the near future to describe traffic behaviour in more detail than present methods.
\end{abstract}

Index Terms-traffic swarm, traffic behaviour, behaviour analysis, game theory, machine learning, deep learning

\section{INTRODUCTION}

$\mathbf{T}$ HE use of transport vehicles has become a part of everyday life for most people. Consequently, in recent decades, the volume and nature of traffic has changed dramatically. It is clear that traffic has become increasingly congested these days, especially on highways and also on the streets of crowded cities. Various vehicles, such as cars, trucks, cyclists, pedestrians, and novel vehicles are competing for the resources of public roads.

In this changing environment, traditional solutions can provide limited results. It is not necessarily economical to increase the amount of resources by building new routes or expanding roads by new lanes. Against this approach, it is necessary to study the more economic exploitation of the existing road resources. As the complexity of transport increases, so does the demand for safer transportation. To ensure safety on roads, a much deeper understanding of traffic situations must be achieved. Soon, with the spreading of higher level automation (or full self-driving capabilities) of vehicles, the predictability of mixed-traffic interactions is necessary to provide safe and efficient algorithms. Besides, an innovative and state-of-theart solution must also meet the needs of comfortably and predictability requirements.

From the perspective of this paper, interactions between vehicles result in actions taken by vehicle drivers. Actions are controlled by behaviour which in turn is influenced by the driver's personality (see Fig. 1). Behaviour is also highly dependent on the context. The same person with a specific personality can behave differently in the same situation but in a different context. Basically, behaviour and personality are

Department of Telecommunications and Media Informatics, Budapes University of Technology and Economics, email: \{hollosi.gergely, lukovszki. csaba, bancsics.mate,magyar.gabor\}@vik.bme.hu

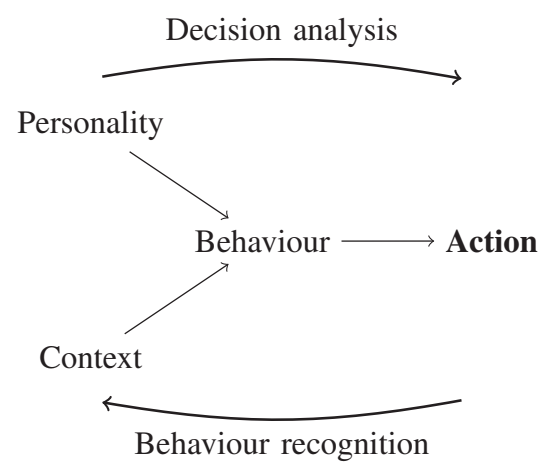

Fig. 1.: The relation of personality, behaviour and action. A specific personality produces an actual behaviour in a peculiar context. The observable actions are derivable from the behaviour.

not observable, so we need to find ways to infer them from actions in specific contexts. For this, two main principles can be identified while analysing traffic behaviour (see Fig. 1). From the point of personality, the behaviour of the driver can be simulated or modelled in common situations; then the behaviour itself or the actions and its consequences can be analysed. This aspect is called decision analysis. In terms of actions, the actual behaviour can be deduced by various techniques, which are called behaviour recognition or identification.

The main goal of this paper is to review and survey research concerning vehicle driver behaviour, especially the ones that describe the behaviour of a vehicle groups called as traffic swarm in this paper. First, in the next section, traffic swarm concept is presented to define the term traffic swarm behaviour. The sections after present the state-of-theart methods of behaviour identification and decision analysis in the literature. The taxonomy of the discussed methods is presented on Figure 2. Finally, the topic is concluded by some important observation and recommendation about future work.

\section{TRAFFIC SWARM}

Social swarms, such as ant colonies, birds, bee hives, flocks, herds, shape group's behaviour through simple decisions of individuals and interactions among them. In recent decades, the research community put focus on researching social swarms in order to understand their collaborative behaviour [1], [2], [3], [4], [5]. As a result of continuous evolution, these swarms have developed intelligent behaviours for survival. The basis of emerging behaviour is formed from the behaviour of individuals and from the communication that takes place among them. The method of communication is different for each species, e.g ants use pheromones to mark their path 


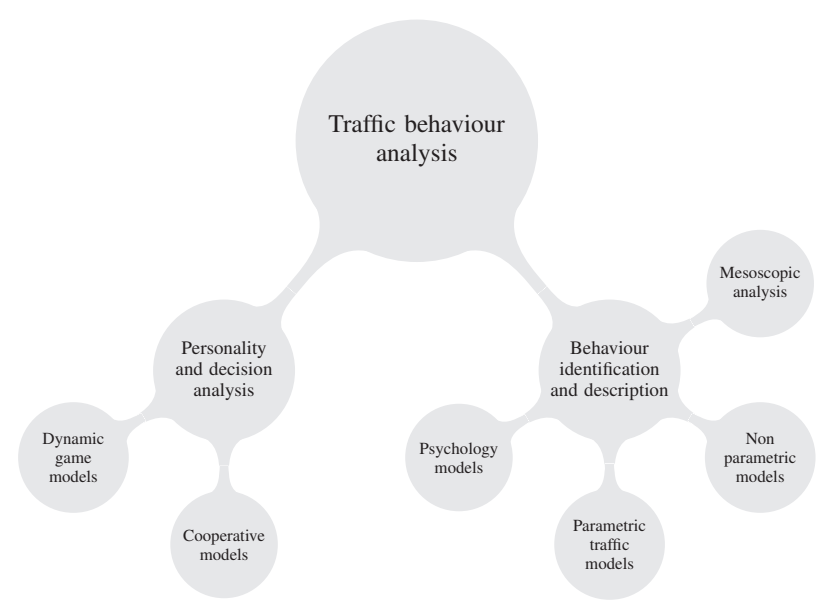

Fig. 2: Overview of the applicable methods of traffic behaviour analysis. The description of the groups and subgroups can be found in the text.

and alert others [2]. This type of communication was first introduced as the concept of stigmergy by Grassé [6]. It was an important step towards understanding the mechanisms of emergence, regulation, and control of collective activities in social insects [6], [7].

On public roads, public transport is also based on collaborative group behaviour. In traditional behaviour modelling, one models an approaching car's breaking as a single event, but in reality this action results in other actions done by the nearby drivers who react to the approaching car. In fact, even the drivers' simplest actions can be described as actions and reactions to each other. This interaction, as stigmergy, relies not only on the distance and the speed of the neighbouring cars, but also affected by the car's movements within the lane, or the observable personal reactions of the driver. Also the resulting action induces reactions of others. This collaborative behaviour is consistent with the behavioural patterns of social swarms.

\section{BEHAVIOUR IDENTIFICATION AND DESCRIPTION}

\section{A. Psychology based models}

The most common and classic way of analysing traffic behaviour is based on psychological behaviour models originally developed in psychological research. These models usually $a$ priori determine a couple of behaviour categories by which the drivers can be characterised and classified. The categories are well known to the human perception, e.g. aggressiveness or cautiousness. Such categories result in a multi-axis (multidimension) space of behaviour patterns, where the dimensions can be described by continuous values, or - typically - by discrete values. For example, [8] uses a 7-point scale to each of the six behaviour categories to express the weight of that behaviour characteristic.

One of the early but exciting development of the cooperation between traffic theory and psychology is the Hierarchical Mental Model (HMM) of the driver [9], which states that the driver behaviour is composed of decision-makers communicating with mental models (see Fig. 3). It divides decision-making into the strategy selection level (e.g. reaching the destination, required travel time, etc.), the tactical level (which selects the

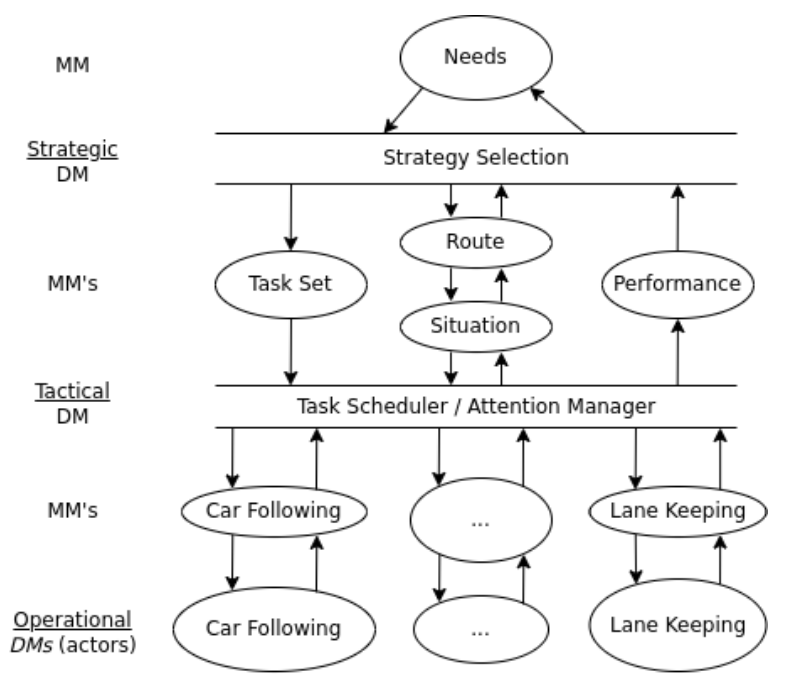

Fig. 3: The Hierarchical Driver Model (HDM). A vehicle driver has different levels of mental models and decision makers. The driver can be described as a couple of mental model running parallel, where each mental model runs at different level. HDM indicates that behaviour is determined not only by tactical decisions but by higher-level strategical decisions. For details, refer to [9].

next manoeuvre or task to perform), and the operational level (common tasks like car following or lane keeping).

The main point is that the observed behaviour can originate from either the tactical (e.g. emergency situation) or strategy selection (e.g. being in a hurry) level. However, in practical scenarios, identifying the mental source is not necessarily, one can readily observe and analyse the behaviour itself.

The usual way of analysing traffic behaviour in psychological models are as follows: first, one defines the behaviour dimensions or latent space axes, then identifies the features that can be observed, maps the features into behavioural latent space and finally, analyses the latent space with various methods or provides future predictions to unseen data.

The most important and also the most studied behavioural characteristic is the degree of aggression which is believed to principally determine the safety of driving [8], [10], [11]. E.g. in [12], aggressiveness is the only dimension studied, and a fuzzy logic method is applied to the observed acceleration to predict the level of aggressiveness on a four-level scale. However, not only aggressiveness can be considered in safety, but the carefulness of the driver is also an important category [13]. Attentive and distracted categories were used by [14], where a couple of observed features (driver pose and environmental factors) were used to predict the nearfuture trajectory of the car by using Convex Markov Chains. The features were processed in a non-supervised manner using k-means algorithm to identify the modes labelled with predefined categories. The DriveSafe application [15] also considers attentiveness and distraction behaviours.

A multidimensional behavioural latent space is defined in [16], [8]. They selected six behaviour dimensions (e.g. aggressiveness, carefulness, etc.) and nine different features; then they conducted surveys to map real-world data onto the six behaviour axes on a 7-point scale. On the latent space, principal component analysis was applied, resulting in the 
conclusion that aggressiveness and carefulness are the main factors behind all the characteristics.

It is worth mentioning that even classic traffic simulation models use psychological categories implicitly or explicitly. For example, the well-known MOBIL [17] lane-changing model uses the politeness factor when making decisions. Car-following models also have hyperparameters that can be described using psychological correspondences. However, the human aspects of the traffic simulation models remain to be researched extensively [18].

\section{B. Parametric traffic models}

The most common methods for analysing microscopic traffic behaviour are based on various parametric models. These representations determine physical, measurable parameters which influence the drivers' behaviour and decision-making process in certain traffic situations. The most common models were developed to describe behaviour in highway traffic, e.g. changing the car's acceleration based on the speed and distance of the vehicle ahead (car-following models, [19], [20], [21]), or the event of lane changing based on the relative speed and available space in the overtaking lane (lane-changing models, [22]). However, more complex models are being developed to describe more complicated events, such as driver behaviour during unexpected lane closures [23].

Almost all parametric models require the specification of some hyperparameters which describe certain driver properties. For example, in the case of the most well-known carfollowing model, namely the Intelligent Driver Model (IDM) [21], a couple of hyperparameters are needed to be defined as inputs of simulations, like desired velocity in free driving, maximum acceleration or comfortable braking deceleration.

The correct setting of these hyperparameters is critical for making the simulation models realistic. This can be done using real traffic data during model calibration, when the input hyperparameters are adjusted in a way that each car's behaviour and trajectory mirrors the real-life data as closely as possible. This optimisation process can be done using analytic calculations [24]; however, newer methods also use artificial intelligence and neural networks [25], [26].

As the IDM example shows, the hyperparameters that describe a chosen vehicle's motion dynamics are constants and can be used to determine the response to different events. However, the value of these hyperparameters also bear useful information and, when they are suited for real-life data, can be used for describing real driver traits [27].

As such, the examination of hyperparameter statistics can be promising. The distribution of these properties can provide useful information about the expectable behaviour of drivers in a given situations, as well as the probability and nature of irregular behaviours [28], which can lead to more accurate predictions [28], [27].

Although these models describe certain driver actions in a realistic and comprehensible way, the understanding of intensive, complex and heterogeneous traffic requires more elaborated methodologies. For example, it may be worth comparing the results of these parametric model analyses with the aforementioned psychological models and finding correlations between the psychological driver traits and the hyperparameters of the trajectories. This can lead to more accurate descriptions of how different types of drivers behave on the road.

\section{Non-parametric machine learning models}

There is a wide literature on the application of nonparametric models to describe driver behaviour in different traffic scenarios. Non-parametric behaviour analysis uses a black-box model. In this machine learning principle, the model learns the driver behaviour from real input and output data. The behaviour itself is the relation between input feature data and the related output response.

The emergence of ADAS (Advanced Driver Assistance System) has emphasised the need and viability of this kind of modelling. In order to provide relevant information to the driver, a system must be capable of understanding what is currently happening at a certain traffic scene. This problem is known as situation assessment [29]. To address this problem, conditional probabilistic Bayesian networks are used to estimate driver intention in road intersections in [30] and [31].

Besides these approaches, machine learning principles are widely used to build non-parametric behaviour models to describe ego driver behaviour. The model input basically is the ego driver measurable relations to its surroundings, the ambient cars, or even static and dynamic signals, signs and lane rules. The common output is the real trajectory of the ego driver's vehicle.

In a typical approach, inputs come from the eight surrounding vehicles, which concept in [32] is extended by the preceding vehicle of the front vehicle resulting in the most reliable behaviour modelling and prediction. Most of the approaches use a full set of input and output data; however, [33] exploited sparse floating car data to predict vehicle trajectories with penetration rate between 2 and 8 percent.

As in many machine learning applications, existing techniques can be split between classification or regression. When applied to motion prediction, classification problems intend to determine a high-level intention, such as lane changing to left or right, lane-keeping or braking during highway driving, turning left, turning right, or going straight in an intersection.

On the other hand, regression problems aim at directly obtaining a predicted future positions of the considered vehicle, which can then be even used for motion planning of target vehicle. Trajectory prediction hypothesises can be used as an input during decision process. ([34])

More recently, the most used approaches for traffic flow prediction are based on deep learning algorithms. The most used methods for traffic prediction are the Long Short-Time Memory (LSTM) and the Generative Adversarial Network (GAN). Because LSTM neural networks are able to keep previous inputs in the memory, they are considered particularly efficient for problems that require learning of patterns in a time series. In [32], authors described a unimodal vehicle trajectory prediction in highway scenes for ADAS systems proposing a Keras framework based LSTM neural network.

However, predicting one manoeuvre is not the best solution. Yuexin et al. [35] showed while studying pedestrian move- 
ments that a person has, couple of choices in a given situation is socially acceptable. This is called multimodal behaviour. Multimodality is highly important: if an algorithm predicts one trajectory only, it can be the average of possible trajectories resulting in a mode collapse. Based on this result, a couple of multimodal solutions were introduced for vehicle manoeuvre prediction, like in [36], [37].

Through intensive analysis [33] proved that, although LSTM-based models are superior in unimodal scenarios, generative models perform best in those where the effects of multimodality are higher. Luca et al. [33] shows that the difference between using an LSTM and a GAN for prediction lies in the objective function. The LSTM learns an average behaviour because it minimizes the average error between all the predictions, while GANs learn to produce plausible samples corresponding to specific behaviours as a result of competition between generator and discriminator networks.

The training phase is essential in machine learning models. Under-represented data usually leads to poor prediction, invalid or unseen situations. This problem of cascading errors [38] is well-known in expert knowledge-based Imitation Learning (IL). Motivated by working on alternative IL methods, [39] proposed Inverse Reinforcement Learning (IRL), implemented with Generative Adversarial Imitation Learning (GAIL) [40]. The model assumes that the expert follows an optimal policy with respect to an unknown reward function. When the reward function is discovered, the model can follow the Reinforcement Learning (RL) method and acts identically as the expert. The proposed imitation even extends the model to unseen scenarios.

In order to estimate the influence of various vehicles near the target vehicle, there is a need to jointly reason and predict the future trajectories of all the vehicles involved in a traffic scene, especially in intersections. In the literature, in most of the scenarios, homogeneous traffic is considered, where although the behaviour can be different, each vehicle or driver follows the same rule. A more demanding situation happens when traffic is heterogeneous. In a heterogeneous scenario, different types of vehicles interact with each other with different behaviour and action sets. This scenario is particularly demanding if the traffic is predominated by smaller vehicles that do not follow lane rules, such as scooters, motorcycles, or bicycles, or dominant vehicles that regulate traffic, such as buses.

To handle heterogeneous traffic, mapping different vehicle types to equivalent homogeneous flow is available in [41], [42]. A more sophisticated solution is proposed in [43]. The authors proposed a conditional GAN model to generate multiple trajectory predictions for vehicles at either signalised or non-signalised intersections.

As a result of building predictive models, latent space is built, which represents the driver behaviour. The explanation of latent space is a fundamental task to understand the learned behaviour. The understanding is based on the traffic scene classification, which traditionally is defined by expert knowledge.

In the literature, different approaches are available for automatic latent space exploration. The goal of [44] was to explain the behaviour of any given black-box classifier instead of just reasoning about its individual predictions. Their explanation relies on the small number of compact decision sets. The authors of [45] introduced human-readable instance-wise feature selection as a methodology for model interpretation. The proposed method learns a function to extract a subset of the most informative features for each given example.

[46] introduced two different unsupervised deep learning approaches to understand and classify real traffic scenes from ADAC ego driver point of view. Authors proved that proposed approaches are capable of learning an expressive latent space for a real-world highway dataset and making scene classification from real datasets.

As a result of explanation, we lose information available in latent space, which is the cost of easier interpretation. Various methods have recently been proposed to help users interpret the predictions of complex models, but it is often unclear how these methods are related to each other and when one method is preferable over another. To address this problem, the authors of [47] present a unified framework for interpreting predictions. Based on the proposed unification, the authors present new methods that show improved computational performance and better consistency with human intuition than previous approaches. Extending the above work, authors show in [44] that a good explanation must follow properties of fidelity, unambiguity, interpretability, and interactivity.

The predictive models' increasing complexity makes it harder to explain or reason the driver behaviour represented in the latent space [48]. It emphasises the need for tools that can explain predictive models' complex behaviour in a faithful and interpretable manner.

\section{Mesoscopic analysis}

There are two common, well-known description strategies for traffic situations: microscopic and macroscopic descriptions. While the latter assigns aggregated measures to the traffic flow, the former describes each vehicle's behaviour individually. However, microscopic description is egoistic, meaning that it views traffic from the point of one vehicle at a time. The interaction between agents (vehicles) is usually simplified to the followed car, and a couple of neighbours defined a priori. The behaviour of the vehicle does not depend directly on the manoeuvres of nearby cars, e.g. the lane change in MOBIL model depends only on the car ahead and the space in the target lane [17]. However, the dependency of the participants is much wider than in the classic microscopic models; in fact, there are a number of vehicles affected by each other mutually. So, we can handle a group of vehicles as a mesoscopic model of traffic situations, where a couple of nearby participants interact with each other continuously. This way, we can analyse the behaviour of this group together, highlighting a deeper relationship between the behaviour of distinct vehicles.

A couple of early works analysed the nature of vehicle groups. In [49] the author found a type of collective behaviour (called ,solid block”) in the case of increasing density of vehicles resulting in a highly coherent state, in which all the vehicles have the same average velocity with little deviation. Besides, [50] showed the closed stability conditions of vehicle 
formations. In contrast, [51] searched for the answer to the question: what characterises a ,free vehicle"? Based on a large number of recorded vehicle movements, she found that time separation is a better measure of freedom than distance-based metrics. There were attempts to discover the conditions of forming and splitting vehicles into groups. In [52], a Bayesian estimator estimated the state of individual vehicles then a density-based clustering approach was applied to identify vehicle groups and group boundaries. They found that the probability of collision is an applicable metric for the closeness of two vehicles.

The latest and most common research in mesoscopic analysis also tries to solve the task of multimodal trajectory and manoeuvre prediction, meaning that not only one trajectory but a couple of possible trajectories are predicted. The topic is extensively researched in automated driving because the behaviour prediction and possible movements of nearby vehicles is a serious safety issue.

After realising that independent traffic models are not successful enough in trajectory and manoeuvre prediction [30], [31], a new theory called ,,social force” was taken over from the analysis of pedestrian behaviour. [53] introduced the term ,,social force" measuring the internal motivations of the individuals to perform certain actions. The social force works like a force in the force field, and is actually a vector, which attracts and distracts: attracts to the desired velocity of motion and keeps a certain distance to other pedestrians. The social force model was improved in order to „learn" the social force from real trajectories which leaded to a so called „,social pooling” layer in LSTM networks [54]. Based on these results, [55] introduced the convolutional social pooling term to consider the social context during trajectory prediction. The social pooling basically learns the spatial interdependencies of the tracks based on the output of LSTM encoders, which we call manoeuvre prediction. The same method was applied in [35], using different participant classes like pedestrians, cars and bicycles. It is worth noting that the behaviour prediction model can be static or dynamic: some works predict fixed manoeuvre classes, others predict graph embeddings or other encodings.

The latest research involves state-of-the-art graph neural networks representing traffic situation as an interaction graph. Interestingly enough, these solutions usually employ the same method: they use a behaviour or manoeuvre prediction model and a trajectory prediction model (see e.g. Fig. 4).

For example, [57], [58] applies a graph convolutional model to extract the dynamic graph features from a graph formed by nearby cars. An LSTM network is then used to generate the trajectory predictions. In [59], the authors use same method, but estimate all the movements of every considered car, and use interaction model based on collision estimates. There are a couple of other methods, using attentive graph networks [60], [61], or joint estimations [56]. Not just vehicle-only interactions, but vehicle-pedestrian interactions were analysed in [62].

These results aim at the topic of trajectory prediction. However, the methods can be made suitable to realise traffic behaviour analysis. Unfortunately, this topic has been rarely

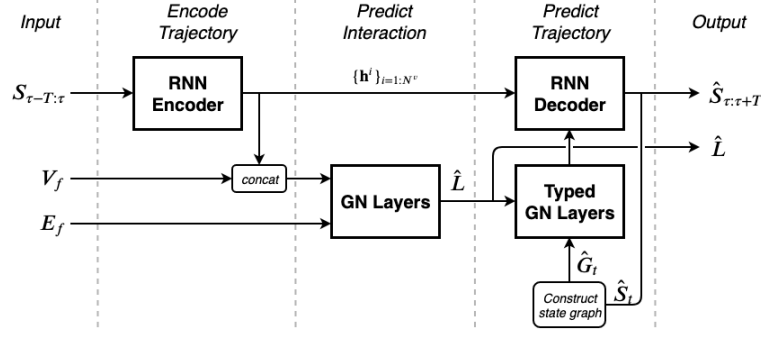

Fig. 4: A typical graph based on trajectory prediction method [56]. These models consist of an interaction prediction part creating features to describe interaction between participants and a trajectory prediction part estimating the trajectories of the participants based on the interaction features. The interac- tion prediction part on this model is readily identifiable which potentially provides internal features to analyse behaviour.

studied, although reasoning in machine learning systems is well-known and widely practiced.

The analysis of the characteristics of traffic behaviour requests the interpretation of the presented machine learning models. A couple of proposed methods can be found in the literature. The main goal is to interpret the behaviour prediction model, which is a graph neural network. Analysing the graph helps to discover the reasons for a selected manoeuvre as a dependence of the neighbouring vehicles. To understand which parts of the graph are contributing to a prediction, [63] proposed an edge classifier that predicts if an edge can be dropped. This way, the most influential interacting parties can be found effectively. Similarly, [64] finds a smaller subgraph and a subset of features that have a crucial role in predictions.

\section{Personality And Decision Analysis}

While identifying and predicting behaviour from observations are widely studied topics, analysing the effect of different personalities and drivers' decisions on traffic is also promising. Personality and decision analysis deals with the interaction of vehicle drivers trying to solve traffic situations mutually. Practical methods apply traffic simulations to resolve the issues of a selected situation; however, they sometimes lack behaviour or personality aspects. Fortunately, we can find game theory models developed to analyse these aspects and select the proper behaviour or action to solve the traffic situation effectively.

The behaviour of the interacting drivers evolves from the personality and the actual situation. This way, the methods typically examined define some traffic situation (e.g. a lane change or a road crossing) and a couple of personality or behaviour patterns. The traffic interactions have a couple of safety, sociological, and communication perspectives. The most complete definition and taxonomy of traffic interaction can be found in [65], which states that traffic interaction is a ,situation where the behaviour of at least two road users can be interpreted as being influenced by the possibility that they are both intending to occupy the same region of space at the same time in the near future". These interactions include not only interactions between vehicle drivers but also, for example, between a vehicle driver and a pedestrian.

Game-theoretic models are widely used in traffic theory, not only in behaviour analysis. For instance, several methods can 
be found for planning the traffic infrastructure in urban spaces [66] or to optimise traffic throughput [67], [68], [69]. However, the first mention of the game theory applied to traffic behaviour analysis dates back to 1984, when Fisk applied Nash noncooperative and Stackelberg games in transportation problems like intercity passenger travel optimisation and traffic signal optimisation [70]. A huge family of game-theoretic traffic behavioural models analyses the traffic behaviour in the aspect of cooperation. Cooperation seems to contradict evolution and natural selection; however, Nowak showed that evolution could actually lead to cooperation [71]. The main point in these kinds of analysis is that a group of players (called cooperators) take joint actions resulting in collective payoffs, while defectors do not attach to these groups. The methods are commonly based on the cellular automata paradigm. It is worth to point out that the validation of game theory models is not always provided, i.e. the game theory model may not describe an observable behaviour in reality.

Modelling freeway traffic, [72] investigated the evolution of cooperative and defective behaviour in lane change situations. They showed that as the difference between the velocity of cooperators and defectors decreases, the fraction of cooperators increases, i.e. in high-density traffic the drivers start to behave as cooperators. At a non-signalised crossing, it was shown that cooperative drivers improve traffic quality, while defective drivers worsen it [73]. It was also presented that there is some relationship between the density of cars on the road and the desire for drivers to go defective. However, defectiveness is not always a bad thing: using Weibull distribution for describing the generation of defectors, it can be shown that while the existence of defectors reduces the safety of the crossing, it is also beneficial for the capacity of the side road [74]. An interesting defective-cooperative model was developed in [75], where the non-ego vehicles switch between cooperative and defective behaviour in the process of interaction. The main idea is that while the non-ego vehicles' core behaviour is cooperative, they have a short duration of defective behaviour (so-called adversarial time), making the ego vehicle behave carefully, e.g. the driver becomes attentive when sees a pedestrian at the edge of the road. Other interesting methods in this topic can be found in [76], [77], [78], [79].

Of course, there are game-theoretic models beyond cooperative games. For example, [80] presents the Game-Theoretic Social Force Model based on the well-known social model of pedestrians or [81] models 2-to-1 lane junctions as an $\mathrm{N}$ prisoner's dilemma game. The [82] review cites 10 gametheoretic models showing why informal norms of behaviour develop, e.g. giving way to cyclists who do not have priority. There is also some criticism regarding the application of gametheoretic models, e.g. that the Nash equilibrium is not always properly applicable in traffic behaviour [83].

While it is a benefit for a human driver to identify the behaviour of other participants, nowadays, even autonomous vehicles (AV) need to be able to predict the behaviour of human drivers. In the near future, mixed traffic situations are going to be widespread, so action planning in $\mathrm{AVs}$ requires behaviour prediction to be executed for safety and efficiency. The main framework of the behaviour analysis of AV game theory models is the dynamic game model, which essentially is a dynamic model of the steps of the game. The model's state is typically composed of the position, the velocity, and the vehicle's heading [84]. These models' most notable decision policy is the so-called level-k reasoning, where all level- $k$ players make strategic decisions by assuming that all of the other players are level- $(k-1)$. A level-0 player makes no strategic decision, e.g. she reflexively brakes if the followed vehicle is breaking. These models commonly handle a colossal state space and make complex optimisations to produce decisions. Level-k models were successfully applied to freeway situations [85] and also to uncontrolled intersections [86]. However, [87] showed on two large naturalistic datasets that even level-0 behaviour can capture most human driving behaviour. Finally, it is worth mentioning that to help behaviour planning research, [88] created a taxonomy of human interactions in traffic conflicts, including vehiclevehicle and vehicle-pedestrian conflicts.

\section{CONCLUSION}

The current paper outlined different methods for driver behaviour analysis, even in traffic situations with complex interactions (see Table I.). However, classical behaviour analysis - which finds reasons and motivations behind actions - is committed by only psychological models while most research topics target trajectory and interaction prediction. While stateof-the-art machine learning models, latent space exploration techniques and game theory methods are successfully applied to numerous problems (e.g. image processing), it is hard to find a solution which addresses entirely the traffic behaviour analysis. It is tempting to improve the presented methods to be applicable for exploring the behaviour behind visible actions, moreover, to reason the behaviour of groups of vehicles.

The consideration of traffic swarms is really promising, where the interactions among drivers are not limited to the surrounding vehicles and are not bound to kinematic properties, so one can analyse the actions as a result of complex behaviour instead of only trajectories or visible interactions of the vehicles. However, mesoscopic modelling provides a reasonable starting point to this research. Beyond that, further research on behaviour interpretation is a promising direction, but modelling, analysing, and extracting the latent space requires more elaborated measures for driver behaviour evaluation.

\section{ACKNOWLEDGMENT}

The research was supported by the Institute of Transport Sciences (KTI) within the Innovative Mobility Program.

The research was supported by the Ministry of Innovation and Technology NRDI Office within the framework of the Autonomous Systems National Laboratory Program. 
TABLE I

THE SUMMARY OF METHODS FOR TRAFFIC BEHAVIOUR ANALYSIS. THE „METHODOLOGIES” COLUMN SHOWS A COUPLE OF KEYWORDS (THEORIES, TECHNOLOGIES, METHODS, ETC.) WHICH MOSTLY, BUT NOT EXCLUSIVELY, DESCRIBE THE METHOD.

\begin{tabular}{|c|c|c|c|c|}
\hline \multicolumn{2}{|c|}{ Method } & Description & Methodologies & References \\
\hline \multirow{2}{*}{$\begin{array}{l}\text { Personality } \\
\text { and } \\
\text { decision } \\
\text { analysis }\end{array}$} & $\begin{array}{l}\text { Dynamic } \\
\text { game } \\
\text { models }\end{array}$ & $\begin{array}{l}\text { The models define the states of the game and the } \\
\text { state transition functions and analyse the dynamic } \\
\text { progression of the states. }\end{array}$ & $\begin{array}{l}\text { dynamic game theory models, } \\
\text { level-k model }\end{array}$ & {$[84][85][86][87][88]$} \\
\hline & $\begin{array}{l}\text { Cooperative } \\
\text { models }\end{array}$ & $\begin{array}{l}\text { Cooperative models find the consequences of some } \\
\text { discrete behaviours in actual traffic situations using } \\
\text { game theory methods. }\end{array}$ & $\begin{array}{l}\text { game theory, cooperative and } \\
\text { non-cooperative games (zero-sum } \\
\text { games, Stackelberg games) }\end{array}$ & $\begin{array}{l}{[65][66][67][68][69][70]} \\
{[71][72][73][74][75][76]} \\
{[77][78][79][80][81][82]} \\
{[83]}\end{array}$ \\
\hline \multirow{4}{*}{$\begin{array}{l}\text { Behaviour } \\
\text { identifica- } \\
\text { tion and } \\
\text { description }\end{array}$} & $\begin{array}{l}\text { Psychology } \\
\text { models }\end{array}$ & $\begin{array}{l}\text { Psychology models determine a priori behaviour cat- } \\
\text { egories and maps visible actions to these categories. }\end{array}$ & $\begin{array}{l}\text { psychology categories, surveys, } \\
\text { latent space analysis (e.g. PCA) }\end{array}$ & $\begin{array}{l}{[8][9][10][11][12][13][14]} \\
{[15][16][17][18]}\end{array}$ \\
\hline & $\begin{array}{l}\text { Parametric } \\
\text { traffic } \\
\text { models }\end{array}$ & $\begin{array}{l}\text { Parametric traffic models are simulation models used } \\
\text { by traffic engineers. The latent space of hyperparam- } \\
\text { eters can be analysed as a behaviour space. }\end{array}$ & $\begin{array}{l}\text { traffic simulation models (e.g. } \\
\text { Wiedemann, IDM), latent } \\
\text { (hyperparameter) space analysis }\end{array}$ & $\begin{array}{l}{[19][20][21][22][23][89]} \\
{[24][25][26][28][27]}\end{array}$ \\
\hline & $\begin{array}{l}\text { Non } \\
\text { parametric } \\
\text { models }\end{array}$ & $\begin{array}{l}\text { Non parametric models are machine learning models } \\
\text { which are mapping visible actions to latent behaviour } \\
\text { spaces. The methodologies are typically based on } \\
\text { generative deep learning methods or non-supervised } \\
\text { learning methods. }\end{array}$ & $\begin{array}{l}\text { trajectory prediction, machine } \\
\text { learning models, deep learning } \\
\text { models (GAN, LSTM), } \\
\text { reinforcement learning, model } \\
\text { explanation }\end{array}$ & $\begin{array}{l}{[29][30][31][32][33][34]} \\
{[35][38][39][40][43][44]} \\
{[45][46][47][48]}\end{array}$ \\
\hline & $\begin{array}{l}\text { Mesoscopic } \\
\text { models }\end{array}$ & $\begin{array}{l}\text { Mesoscopic models are between microscopic and } \\
\text { macroscopic models. They are commonly using } \\
\text { graph based solutions to analyse complex interac- } \\
\text { tions among participants. }\end{array}$ & $\begin{array}{l}\text { interaction prediction, social force } \\
\text { models, social pooling, graph } \\
\text { neural networks, model } \\
\text { explanation }\end{array}$ & $\begin{array}{l}{[17][49][50][51][52][30]} \\
{[31][54][55][35][53][57]} \\
{[58][59][60][61][56][62]} \\
{[63][64][44][90][45]}\end{array}$ \\
\hline
\end{tabular}

\section{REFERENCES}

[1] E. Bonabeau, G. Theraulaz, J.-L. Deneubourg, S. Aron, and S. Camazine, "Self-organization in social insects," Trends in Ecology and Evolution, vol. 12, p. 188, 05 1997, DoI: 10.1016/S0169-5347(97)01048-3.

[2] P. Karlson and M. Lüscher, "Pheromones: a new term for a class of biologically active substances," Nature, vol. 183, pp. 55-56, 011959 , DOI: $10.1038 / 183055 \mathrm{a} 0$.

[3] G. Theraulaz, E. Bonabeau, S. C. Nicolis, R. V. Solé, V. Fourcassié, S. Blanco, R. Fournier, J.-L. Joly, P. Fernández, A. Grimal, P. Dalle, and J.-L. Deneubourg, "Spatial patterns in ant colonies," Proceedings of the National Academy of Sciences, vol. 99, no. 15, pp. 9645-9649, 2002. [Online]. Available: https://www.pnas.org/content/99/15/9645. DoI: $10.1073 /$ pnas. 152302199

[4] K. von Frisch, "The dance language and orientation of bees," 1967," DoI: 10.4159 /harvard.9780674418776.

[5] C. W. Reynolds, "Flocks, herds and schools: A distributed behavioral model," in Proceedings of the 14th Annual Conference on Computer Graphics and Interactive Techniques, ser. SIGGRAPH '87. New York, NY, USA: Association for Computing Machinery, 1987, p. 25-34, DoI: $10.1145 / 37401.37406$.

[6] P.-P. Grasse, "La reconstruction du nid et les coordinations interindividuelles chez bellicositermes natalensis et cubitermes sp. la theorie de la stigmergie: Essai d'interpretation du comportement des termites constructeurs." Insectes Sociaux, vol. 6, pp. 41-81, 1959

[7] G. Theraulaz and E. Bonabeau, "A brief history of stigmergy," Artificial life, vol. 5, pp. 97-116, 02 1999, DoI: 10.1162/106454699568700.

[8] E. Cheung, A. Bera, E. Kubin, K. Gray, and D. Manocha, "Identifying driver behaviors using trajectory features for vehicle navigation," 2018,” DoI: 10.1109/IROS.2018.8594348.

[9] E. R. Boer and D. M. Hoedemaeker, "Modeling driver behaviour with different degrees of automation: a hierarchical decision framework of interacting mental models," 1998.

[10] P. Harris, J. Houston, J. Vazquez, J. Smither, A. Harms, J. Dahlke, and D. Sachau, "The prosocial and aggressive driving inventory (padi): A self-report measure of safe and unsafe driving behaviors," Accident Analysis and Prevention, vol. 72, p. 1-8, 112014 , DoI: 10.1016/j.aap.2014.05.023.

[11] F. Zhong-xiang, L. Yang-yang, L. Jing, and Z. Wei-hua, "Selectedmodel and sensitivity analysis of aggressive driving behavior," China Journal of Highway and Transport, vol. 25, no. 2, pp. 106-112, 2012.
[12] A. Aljaafreh, N. Alshabatat, and M. S. Najim Al-Din, "Driving style recognition using fuzzy logic," in 2012 IEEE International Conference on Vehicular Electronics and Safety (ICVES 2012), 2012, pp. 460-463, DOI: 10.1109/ICVES.2012.6294318.

[13] R. Vasudevan, V. Shia, Y. Gao, R. Cervera-Navarro, R. Bajcsy, and F Borrelli, "Safe semi-autonomous control with enhanced driver modeling," in 2012 American Control Conference (ACC), 2012, pp. 28962903, DoI: 10.1109/ACC.2012.6315654.

[14] D. Sadigh, K. Driggs-Campbell, A. Puggelli, W. Li, V. Shia, R. Bajcsy, A. Vincentelli, S. Sastry, and S. Seshia, "Data-driven probabilistic modeling and verification of human driver behavior," 032014.

[15] L. M. Bergasa, D. Almería, J. Almazán, J. J. Yebes, and R. Arroyo, "Drivesafe: An app for alerting inattentive drivers and scoring driving behaviors," in 2014 IEEE Intelligent Vehicles Symposium Proceedings, 2014, pp. 240-245, DoI: 10.1109/IVS.2014.6856461.

[16] A. Bera and D. Manocha, "Behavior modeling for autonomous driving," in Proceedings of the AAAI Fall Symposium on Reasoning and Learning in Real-World Systems for Long-Term Autonomy, 2018, pp. 16-21.

[17] A. Kesting, M. Treiber, and D. Helbing, "General lane-changing model mobil for car-following models," Transportation Research Record, vol. 1999, pp. 86-94, 01 2007, DoI: 10.3141/1999-10.

[18] M. Saifuzzaman and Z. Zheng, "Incorporating human-factors in carfollowing models: A review of recent developments and research needs," Transportation Research Part C: Emerging Technologies, vol. 48, pp. 379-403, 2014. [Online]. Available: https://www.sciencedirect. com/science/article/pii/S0968090X14002551. DoI: $10.1016 / j . t r c .2014 .09 .008$

[19] R. Wiedemann, "Simulation des strassenverkehrsflusses." 1974.

[20] P. Gipps, "A behavioural car-following model for computer simulation," Transportation Research Part B: Methodological, vol. 15, no. 2, pp. 105-111, 1981. [Online]. Available: h t t p s : //w w w. sciencedirect.com/science/article/pii/0191261581900370. DoI: $10.1016 / 0191-2615(81) 90037-0$

[21] M. Treiber, A. Hennecke, and D. Helbing, "Congested traffic states in empirical observations and microscopic simulations," Phys. Rev. E, vol. 62, pp. 1805-1824, Aug 2000, Dor: 10.1103/PhysRevE.62.1805.

[22] M. Brackstone, M. McDonald, and J. Wu, "Lane changing on the motorway: factors affecting its occurrence, and their implications," IET Conference Proceedings, pp. 160-164(4), January 1998, DOI: $10.1049 / \mathrm{cp}: 19980175$. 
[23] B. Mahmood and J. Kianfar, "Driver behavior models for heavy vehicles and passenger cars at a work zone," Sustainability, vol. 11, no. 21, 2019. [Online]. Available: https://www.mdpi.com/2071-1050/ 11/21/6007. DoI: $10.3390 /$ su11216007

[24] C. Chen, L. Li, J. Hu, and C. Geng, "Calibration of mitsim and idm carfollowing model based on ngsim trajectory datasets," in Proceedings of 2010 IEEE International Conference on Vehicular Electronics and Safety, 2010, pp. 48-53, DoI: 10.1109/ICVES.2010.5550943.

[25] I. I. Otković, T. Tollazzi, and M. Šraml, "Calibration of microsimulation traffic model using neural network approach," Expert Systems with Applications, vol. 40, no. 15, pp. 5965-5974, 2013. [Online]. Available: https://www.sciencedirect.com/science/article/ pii/ S0957417413002893. DoI: 10.1016/j.eswa.2013.05.003

[26] P. Fan, J. Guo, H. Zhao, J. S. Wijnands, and Y. Wang, "Car-following modeling incorporating driving memory based on autoencoder and long short-term memory neural networks," Sustainability, vol. 11, no. 23, 2019. [Online]. Available: https://www.mdpi.com/2071-1050/ 11/23/6755. DoI: $10.3390 /$ su 11236755

[27] B. Hegde, M. O'Keefe, S. Muldoon, J. Gonder, and C.-F. Chang, "Real-world driving features for identifying intelligent driver model parameters," 4 2021. [Online]. Available: https://www.osti.gov/biblio/1779054. DoI: 10.4271/2021-01-0436

[28] M. Pourabdollah, E. Bjärkvik, F. Fürer, B. Lindenberg, and K. Burgdorf, "Calibration and evaluation of car following models using realworld driving data," in 2017 IEEE 20th International Conference on Intelligent Transportation Systems (ITSC), 2017, pp. 1-6, DOI: $10.1109 /$ ITSC.2017.8317836.

[29] M. Endsley, "Theoretical underpinnings of situation awareness: A critical review," Situation awareness analysis and measurement, pp. 3-32, Jan 2000, DoI: 10.1201/B12461-7.

[30] S. Lefèvre, C. Laugier, and J. Ibañez-Guzmán, "Exploiting map information for driver intention estimation at road intersections," in 2011 IEEE Intelligent Vehicles Symposium (IV), 2011, pp. 583-588, DOI: $10.1109 /$ IVS.2011.5940452.

[31] J. Firl, H. Stu "bing, S. A. Huss, and C. Stiller, "Predictive ma- neuver evaluation for enhancement of car-to-x mobility data," in 2012 IEEE Intelligent Vehicles Symposium, 2012, pp. 558-564, DoI: $10.1109 /$ IVS.2012.6232217.

[32] F. Altché and A. de La Fortelle, "An 1stm network for highway trajectory prediction," in 2017 IEEE 20th International Conference on Intelligent Transportation Systems (ITSC). IEEE, Oct 2017. DoI: $10.1109 /$ itsc.2017.8317913.

[33] L. Rossi, A. Ajmar, M. Paolanti, and R. Pierdicca, "Vehicle trajectory prediction and generation using 1 stm models and gans," PLOS ONE, vol. 16, no. 7, pp. 1-28, Jul 2021, DoI: $10.1371 /$ journal.pone. 0253868

[34] D. Dolgov, "Google self-driving car project monthly report," Google Inc., Tech Report, Feb 2016.

[35] Y. Ma, X. Zhu, S. Zhang, R. Yang, W. Wang, and D. Manocha, "Trafficpredict: Trajectory prediction for heterogeneous traffic-agents," 2019," arXiv:1811.02146.

[36] N. Deo and M. M. Trivedi, "Multi-modal trajectory prediction of surrounding vehicles with maneuver based lstms," 2018 IEEE Intelligent Vehicles Symposium (IV), Jun 2018, DOI: 10.1109 /ivs.2018.8500493.

[37] H. Cui, V. Radosavljevic, F.-C. Chou, T.-H. Lin, T. Nguyen, T.-K. Huang, J. Schneider, and N. Djuric, "Multimodal trajectory predictions for autonomous driving using deep convolutional networks," 2019," DOI: 10.1109/ICRA.2019.8793868.

[38] S. Ross and D. Bagnell, "Efficient reductions for imitation learning," in 13th International Conference on Artificial Intelligence and Statistics, ser. Machine Learning Research, Y. W. Teh and M. Titterington, Eds., vol. 9, no. 5. Chia Laguna Resort, Sardinia, Italy: PMLR, May 2010, pp. 661-668.

[39] A. Kuefler, J. Morton, T. Wheeler, and M. Kochenderfer, "Imitating driver behavior with generative adversarial networks," in Intelligent Vehicles Symposium (IV). IEEE, Jun 2017. DOI: 10.1109 /ivs.2017.7995721.

[40] J. Ho and S. Ermon, "Generative adversarial imitation learning," in 30th International Conference on Neural Information Processing Systems (NIPS). ACM, Oct 2016, pp. 4572-4580, arXiv: 1606.03476.
[41] X. Liang, Z. Liu, and K. Qian, "Capacity analysis of signalized intersections under mixed traffic conditions," Journal of Transportation Systems Engineering and Information Technology, vol. 11, no. 2, pp. 91-99, Apr 2011, DoI: 10.1016/S1570-6672(10)60116-X.

[42] N. Cao and K. Sano, "Estimating capacity and motorcycle equivalent units on urban roads in hanoi, vietnam," Journal of Transportation Engineering, vol. 138, no. 6, pp. 776-785, 2012, DOI: $10.1061 /($ ASCE)TE.1943-5436.0000382.

[43] D. Roy, T. Ishizaka, C. K. Mohan, and A. Fukuda, "Vehicle trajectory prediction at intersections using interaction based generative adversarial networks," in Intelligent Transportation Systems Conference (ITSC), Oct 2019, pp. 2318-2323, DOI: 10.1109/ITSC.2019.8916927.

[44] H. Lakkaraju, E. Kamar, R. Caruana, and J. Leskovec, "Interpretable \& explorable approximations of black box models," 2017," arXiv: 1707.01154

[45] J. Chen, L. Song, M. J. Wainwright, and M. I. Jordan, "Learning to explain: An information-theoretic perspective on model interpretation," 2018," arXiv:1802.07814.

[46] N. Harmening, M. Bilos`, and S. Günnemann, "Deep representation learning and clustering of traffic scenarios," 2020," arXiv:2007.07740.

[47] S. Lundberg and S.-I. Lee, "A unified approach to interpreting model predictions," in 31st International Conference on Neural Information Processing Systems. ACM, Dec 2017, pp. 4768-4777, arXiv:1705.07874.

[48] M. T. Ribeiro, S. Singh, and C. Guestrin, "'why should i trust you?": Explaining the predictions of any classifier," in 22nd ACM SIGKDD International Conference on Knowledge Discovery and Data Mining, Aug 2016, pp. 1135-1144, DoI: 10.1145/2939672.2939778.

[49] D. Helbing and B. Huberman, "Coherent moving states in highway traffic," Nature, vol. 396, pp. 738-740, 12 1998, DoI: 10.1038/25499.

[50] J. A. Fax and R. M. Murray, "Graph laplacians and stabilization of vehicle formations," IFAC Proceedings Volumes, vol. 35, no. 1, pp. 55-60, 2002, 15th IFAC World Congress. [Online]. Available: https://www.sciencedirect.com/science/article/pii/S1474667015385116. DOI: $10.3182 / 20020721-6-E S-1901.00090$

[51] K. Vogel, "What characterizes a "free vehicle" in an urban area?" Transportation Research Part F: Traffic Psychology and Behaviour, vol. 5, no. 1, pp. 15-29, 2002. [Online]. Available: https://www. sciencedirect.com/science/article/pii/S1369847802000037. DoI: $10.1016 / \mathrm{S} 1369-8478(02) 00003-7$

[52] Q. Wang and B. Ayalew, "A probabilistic framework for tracking the formation and evolution of multi-vehicle groups in public traffic in the presence of observation uncertainties," IEEE Transactions on Intelligent Transportation Systems, vol. 19, no. 2, pp. 560-571, 2018, DOI: 10.1109/TITS.2017.2750073.

[53] D. Helbing and P. Molnár, "Social force model for pedestrian dynamics," Physical Review E, vol. 51, no. 5, p. 4282-4286, May 1995, DoI: $10.1103 /$ physreve.51.4282.

[54] A. Alahi, K. Goel, V. Ramanathan, A. Robicquet, L. Fei-Fei, and S. Savarese, "Social lstm: Human trajectory prediction in crowded spaces," in 2016 IEEE Conference on Computer Vision and Pattern Recognition (CVPR), 2016, pp. 961-971, DoI: 10.1109/CVPR.2016.110.

[55] N. Deo and M. M. Trivedi, "Convolutional social pooling for vehicle trajectory prediction,” 2018,” DoI: 10.1109/CVPRW.2018.00196.

[56] D. Lee, Y. Gu, J. Hoang, and M. Marchetti-Bowick, "Joint interaction and trajectory prediction for autonomous driving using graph neural networks," 2019," arXiv:1912.07882.

[57] X. Li, X. Ying, and M. C. Chuah, "Grip: Graph-based interactionaware trajectory prediction," in 2019 IEEE Intelligent Transportation Systems Conference (ITSC), 2019, pp. 3960-3966, DoI: $10.1109 /$ ITSC.2019.8917228.

[58] _ _ "Grip++: Enhanced graph-based interaction-aware trajectory prediction for autonomous driving," 2020," DoI: $10.13140 /$ RG.2.2.16886.63044

[59] E. Jo, M. Sunwoo, and M. Lee, "Vehicle trajectory prediction using hierarchical graph neural network for considering interaction among multimodal maneuvers," Sensors, vol. 21, no. 16, 2021. [Online]. Available: https://www.mdpi.com/1424-8220/21/16/5354. DoI: $10.3390 / \mathrm{s} 21165354$

[60] A. Monti, A. Bertugli, S. Calderara, and R. Cucchiara, "Dag-net: Double attentive graph neural network for trajectory forecasting," 2020," DOI: 10.1109/ICPR48806.2021.9412114.8 
[61] J. Li, H. Ma, Z. Zhang, and M. Tomizuka, "Social-wagdat: Interactionaware trajectory prediction via wasserstein graph double-attention network," 2020," DoI: 10.13140/RG.2.2.25253.04320.

[62] Z. Li, J. Gong, C. Lu, and Y. Yangtian, "Interactive behavior prediction for heterogeneous traffic participants in the urban road: A graphneural-network-based multitask learning framework," IEEE/ASME Transactions on Mechatronics, vol. PP, p. 1, 03 2021, DOI: 10.1109/TMECH.2021.3073736.

[63] M. S. Schlichtkrull, N. D. Cao, and I. Titov, "Interpreting graph neural networks for nlp with differentiable edge masking," 2021," arXiv:2010.00577.

[64] R. Ying, D. Bourgeois, J. You, M. Zitnik, and J. Leskovec, "Gnnexplainer: Generating explanations for graph neural networks," 2019," DOI: $10.5555 / 3454287.3455116$.

[65] G. Markkula, R. Madigan, D. Nathanael, E. Portouli, Y. M. Lee, A Dietrich, J. Billington, A. Schieben, and N. Merat, "Defining interactions: a conceptual framework for understanding interactive behaviour in human and automated road traffic," Theoretical Issues in Ergonomics Science, vol. 21, no. 6, pp. 728-752, 2020, DoI: $10.1080 / 1463922 X .2020 .1736686$.

[66] S. Sakaue and K. Nakamura, "Differentiable equilibrium computation with decision diagrams for stackelberg models of combinatorial congestion games," 2021," arXiv:2110.01773.

[67] V. Morandi, "Bridging the user equilibrium and the system optimum in static traffic assignment: how the cooperation among drivers can solve the congestion problem in city networks," 2021," arXiv:2105.05804.

[68] H. Heaton, D. McKenzie, Q. Li, S. W. Fung, S. Osher, and W. Yin, "Learn to predict equilibria via fixed point networks," 2021," arXiv:2106.00906.

[69] L. Cianfanelli, G. Como, A. Ozdaglar, and F. Parise, "Optimal intervention in traffic networks," 2021," arXiv:2102.08441.

[70] C. Fisk, "Game theory and transportation systems modelling," Transportation Research Part B: Methodological, vol. 18, no. 4, pp. 301- 313, 1984. [Online]. Available: https://www.sciencedirect.com/ science/article/abs/pii/0191261584900134. DOI: 10.1016/0191-2615(84)90013-4

[71] M. A. Nowak, "Five rules for the evolution of cooperation," Science, vol 314, no. 5805, pp. 1560-1563, 2006, Dor: 10.1126/science.1133755.

[72] L. E. Cortés-Berrueco, C. Gershenson, and C. R. Stephens, "Traffic games: Modeling freeway traffic with game theory," PLOS ONE, vol 11, no. 11, pp. 1-34, 11 2016, Dor: 10.1371/journal.pone.0165381.

[73] K. J. Szajowski and K. Włodarczyk, "Drivers' skills and behavior vs traffic at intersections," Mathematics, vol. 8, no. 3, p. 433, Mar 2020, DoI: $10.3390 /$ math 8030433

[74] H. Fan, B. Jia, J. Tian, and L. Yun, "Characteristics of traffic flow at a non-signalized intersection in the framework of game theory," Physica A: Statistical Mechanics and its Applications, vol. 415, pp. 172- 180, 2014. [Online]. Available: https://www.sciencedirect.com/science/ article/pii/S0378437114006001. Dor: 10.1016/j.physa.2014.07.031

[75] C.-Y. Chiu, D. Fridovich-Keil, and C. J. Tomlin, "Encoding defensive driving as a dynamic nash game," 2021," arXiv:2011.04815.

[76] L. Sun, M. Cai, W. Zhan, and M. Tomizuka, "A game-theoretic strategyaware interaction algorithm with validation on real traffic data," in 2020 IEEE/RSJ International Conference on Intelligent Robots and Systems (IROS), 2020, pp. 11038-11044, DOI: 10.1109/IROS45743.2020.9340988.

[77] P. Geiger and C.-N. Straehle, "Learning game-theoretic models of multiagent trajectories using implicit layers," 2021," arXiv:2008.07303.

[78] X. Liao, X. Zhao, G. Wu, M. Barth, Z. Wang, K. Han, and P. Tiwari, "A game theory based ramp merging strategy for connected and automated vehicles in the mixed traffic: A unity-sumo integrated platform," 2021," arXiv:2101.11237.

[79] L. Wastavino, B. Toledo, J. Rogan, R. Zarama, V. Muñoz, and J. Valdivia, "Modeling traffic on crossroads," Physica A: Statistical Mechanics and its Applications, vol. 381, pp. 411-419, 2007. [Online]. Available: https://www.sciencedirect.com/science/article/ pii/ S0378437107003330. DoI: 10.1016/j.physa.2007.03.052

[80] F. T. Johora, D. Yang, J. P. Müller, and Ümit Özgüner, "On the generalizability of motion models for road users in heterogeneous shared traffic spaces," 2021," arXiv:2101.06974.

[81] A. Yamauchi, J. Tanimoto, A. Hagishima, and H. Sagara, "Dilemma game structure observed in traffic flow at a 2-to-1 lane junction," Physical review. E, Statistical, nonlinear, and soft matter physics, vol. 79, p. 036104, 04 2009, DoI: 10.1103/PhysRevE.79.036104.
[82] R. Elvik, "A review of game-theoretic models of road user behaviour," Accident Analysis \& Prevention, vol. 62, pp. 388-396, 2014. [Online]. Available: https://www.sciencedirect.com/science/article/ pii/ S0001457513002443. Dor: 10.1016/j.aap.2013.06.016

[83] J. Wright and K. Leyton-Brown, "Beyond equilibrium: Predicting human behavior in normal-form games," vol. 2, 07 2010, DOI: $10.1145 / 1807406.1807449$.

[84] J. F. Fisac, E. Bronstein, E. Stefansson, D. Sadigh, S. S. Sastry, and A. D. Dragan, "Hierarchical game-theoretic planning for autonomous vehicles," 2018," arXiv: 1810.05766

[85] N. Li, D. Oyler, M. Zhang, Y. Yildiz, I. Kolmanovsky, and A. Girard, "Game-theoretic modeling of driver and vehicle interactions for verifi- cation and validation of autonomous vehicle control systems," 2016," arXiv: 1608.08589

[86] R. Tian, N. Li, I. Kolmanovsky, Y. Yildiz, and A. Girard, "Game-theoretic modeling of traffic in unsignalized intersection network for autonomous vehicle control verification and validation," 2020," arXiv:1910.07141.

[87] A. Sarkar, K. Larson, and K. Czarnecki, "Generalized dynamic cognitive hierarchy models for strategic driving behavior," 2021," arXiv: 2109.09861.

[88] _ _ "A taxonomy of strategic human interactions in traffic conflicts," 2021," arXiv:2109.13367.

[89] M. Treiber and A. Kesting, Traffic flow dynamics. Springer, 2013. DoI: $10.1007 / 978-3-642-32460-4$

[90] D. Neil, J. Briody, A. Lacoste, A. Sim, P. Creed, and A. Saffari, "Interpretable graph convolutional neural networks for inference on noisy knowledge graphs," 2018," arXiv:1812.00279.

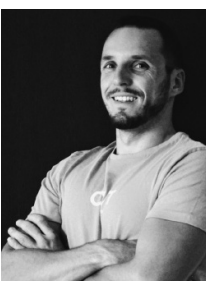

Gergely Hollósi is a researcher at Dept. of Telecommunications and Media Informatics (TMIT) of Budapest University of Technology and Economics (BME). Gergely received his M.Sc. in the Budapest University of Technology and Economics (BME) in 2009. He is actively working and researching on computer vision, image processing, machine learning algorithms and indoor localization systems.

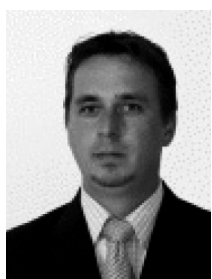

Csaba Lukovszki is a researcher at Budapest University of Technology and Economics (BME). He is technical leader of $\mathrm{RnD}$ projects, has courses in areas of telecommunications, radio- and vision-based indoor localization, and machine learning algorithms. $\mathrm{He}$ is author of 10 journals and 25 conference papers. He is a member of Scientific Association for Infocomm. in Hungary, from he received one Silver and two Gold medals. For a significant publication he is a proud owner of Pollák-Virág Prize.

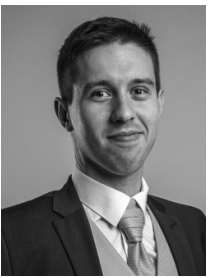

Máté Bancsics is a researcher Dept. of Telecommunications and Media Informatics (TMIT) at Budapest University of Technology and Economics (BME). Máté got his Master's Degree in Electrical Engineering at the same institute in 2021 . He is actively working and doing research on Indoor Positioning Systems, Internet of Things and Machine Learning Algorithms.

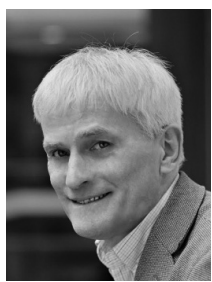

Gábor Magyar is Associated Professor at BME Dept. of Telecommunications and Media Informatics. Head of Data Management Working Group in the Hungarian Mobility Platform, Head of Data Management and Communications in Autonomous Systems National Laboratory, Head of Data Management and Communications at AutomatedDrive Lab. Adviser to the National Council for Infocommunications to the Hungarian Government. Honorary President of the Scientific Association for Infocommunications (HTE), and President of the Hungarian Oracle User Group (HOUG). Served as the Director of Stategic Affaires in BME, and ministerial commissioner for European Institute of Technology and Innovation. He is the recipient of the Kalmár László, Pollák-Virág and Puskás Tivadar awards. 